\title{
The Co Relation of Serum Uric Acid with Risk Factors and Severity of Coronary Artery Disease (CAD) in Acute Coronary Syndrome
}

\author{
Jisa George ${ }^{1}$, SP Kataria², HS Isser ${ }^{3}$ \\ ${ }^{1}$ Assistant Professor, Department of General Medicine, ESIMC \& PGIMSR, Chennai, ${ }^{2}$ Associate Professor, Department of \\ General Medicine, HOD, Department of Medical Oncology, VMMC \& Safdarjung Hospital, New Delhi, ${ }^{3}$ Professor, Department \\ of Cardiology, VMMC \& Safdarjung Hospital, New Delhi, India
}

Corresponding author: Jisa George, Assistant Professor, Department of General Medicine, ESIMC \& PGIMSR, Chennai, India

DOI: http://dx.doi.org/10.21276/ijcmsr.2019.4.4.26

How to cite this article: Jisa George, SP Kataria, HS Isser. The co relation of serum uric acid with risk factors and severity of coronary artery disease (CAD) in acute coronary syndrome. International Journal of Contemporary Medicine Surgery and Radiology. 2019;4(4):D107-D112.

\section{A B S T R A C T}

Introduction: Several epidemiologic studies have shown elevated uric acid levels to predict increased risk of cardiovascular events. Few studies have addressed the value of this biomarker across the whole spectrum of patients with ACS (Acute Coronary Syndrome) especially in Indian settings. Thus, the aim of this study was investigate whether there is an association between serum uric acid level with CAD (Coronary Artery Disease) risk factors its and severity in ACS patients.

Material and methods: This cross -sectional study included 100 consecutive patients admitted with acute coronary syndrome in the cardiology and medicine department of the institution. The presence and severity of CAD was determined by the following clinical vessel score; Absent: no coronary lesions, 1 vessel, 2 vessel and 3 vessel - disease based on the number of coronary arteries involved. The correlation of serum uric acid levels with CAD risk factors and the severity of CAD in the ACS patients was analysed statistically.

Results: There was a significant association between uric acid and the coronary angiographic findings $(P<0.001)$. The patients in the hyperuricemia group had more number of coronary vessels involved than the other group.

Conclusion: The serum uric acid level is correlated with the presence and severity of CAD by coronary angiographic findings $(P<0.001)$ in the acute coronary syndrome patients. Uric acid being an inexpensive and easily available biochemical marker, it will be of much use in settings with limited resources. Serum uric acid may be used to predict the severity of CAD.

Keywords: Acute Coronary Syndrome, Serum Uric Acid, Severity of CAD (Coronary Artery Disease), Gensini Score

\section{INTRODUCTION}

Acute coronary syndromes (ACS) remain a leading cause of morbidity and mortality worldwide. ${ }^{1}$ Though there have been various risk stratification schemes, ${ }^{2} \mathrm{ACS}$ remain poorly characterized in terms of risk prediction. Uric acid is the end product of purine catabolism in humans and is readily tested in routine clinical practice. Although the possibility of an association between elevated uric acid level and cardiovascular disease has been recognized for more than 130 years, the role of acid uric as a risk factor or a risk marker for cardiovascular disease remains a debatable issue. ${ }^{3}$ The association between elevated uric acid and increased risk for mortality, has been investigated in the general population, ${ }^{4}$ and in congestive heart failure, hypertension, ${ }^{5}$ diabetes, ${ }^{6}$ angiographically confirmed coronary artery disease, ${ }^{7}$ and acute myocardial infarction.

Increased uric acid serum levels are a common finding in patients with high blood pressure, insulin resistance, obesity and cardiovascular disease. Furthermore, uric acid as a $\mathrm{CV}$ risk factor has been addressed in numerous prospective and cohort studies.

Several epidemiologic studies have shown elevated uric acid levels to predict increased risk of cardiovascular events, although lack of an independent relationship has also been found. It is conceivable that the interpretation of the "independent" role of uric acid is further complicated by the use of diuretics and by the very close correlation of uric acid levels with established cardiovascular risk factors such as hypertension, obesity, low levels of high-density lipoprotein cholesterol, hypertriglyceridemia, hyperinsulinemia, and reduced insulin sensitivity, all of which are components of the metabolic or insulin resistance syndrome. Moreover, there are other features of atherosclerosis, such as inflammation, oxidative stress, and endothelial dysfunction, that have also been associated with increased serum uric acid levels. Furthermore, clinical ischemic heart, cerebrovascular, and even mild renal disease are all associated with increased uric acid levels.

Few studies have addressed the value of this biomarker across the whole spectrum of patients with ACS (acute coronary syndrome) especially in Indian settings. Thus, the aim of 
this study was to assess the uric acid levels in ACS patients and to investigate whether there is an association between serum uric acid level with CAD (coronary artery disease) risk factors and severity of CAD in ACS patients.

\section{MATERIAL AND METHODS}

The study was conducted in the Department of Medicine and Department of Cardiology, VMMC and Safdarjang Hospital, New Delhi in 100 consecutive patients admitted with diagnosis of acute coronary syndrome (ACS) from January 2013 to December 2013. Ethics committee approval was obtained before the start of the study by the Institutional Ethics Committee. Written informed consent was obtained from each participant or their legal representatives during hospitalization.

The patients selected were consecutive patients admitted in the Cardiology and General Medicine department with Acute Coronary Syndrome, which included patients with ST segment elevation myocardial infarction (STEMI), Non ST segment elevation myocardial infarction (NSTEMI) \& UNSTABLE ANGINA and those undergoing coronary angiography. All patients were given standard treatment of acute coronary syndrome as advised by the treating cardiologist. The patients with the following conditions were excluded:

impaired renal function (Serum Creatinine $>1.5 \mathrm{mg} / \mathrm{dl}$ ), known cases of malignancies, gout or other inflammatory diseases, those using steroids/cytotoxic drugs, patients on drugs which increase serum uric acid e.g.salicylates (>2 gm/d), diuretics, ethambutol, pyrazinamide etc.anti-oxidants, as well as those taking medications targeted to lower uric acid levels and those not undergoing coronary angiography.

\section{Methodology}

Based on the criteria patients were selected and all subjects underwent detailed history and clinical examination. Blood samples were collected next morning for examination on an empty stomach from each patient for Serum uric acid and the lipid profile. The following values were considered normal: total cholesterol $<200 \mathrm{mg} / \mathrm{dl}$, serum triglyceride < $150 \mathrm{mg} / \mathrm{dl}$, serum HDL-C < 40mg/dl and serum LDL < 100 $\mathrm{mg} / \mathrm{dl}$. Patients with hyperu $\neg$ ricemia were defined as serum uric acid concentrations $\geq 7.0 \mathrm{mg} / \mathrm{dL}$ in men and $\geq 6.0 \mathrm{mg} /$ $\mathrm{dL}$ in women. Patient also underwent the following baseline investigations: $\mathrm{CBC}$, Blood sugar levels, renal function test, serum electrolytes and cardiac enzymes (eg.Trop I,CPK$\mathrm{MB})$. The presence and severity of CAD was determined by the following clinical vessel score: Absent: no coronary lesions, 1 vessel, 2 vessel and 3 vessel - disease based on the number of coronary arteries involved. The degree of stenosis was defined as the greatest percentage reduction of luminal diameter in any view compared with the nearest normal segment and was determined visually.

Patients were also evaluated for the following conventional CAD risk factors: Cigarette smoking Diabetes mellitus, Hypertension Low high-density lipoprotein (HDL) cholesterol level: $<1.0 \mathrm{mmol} / \mathrm{L}(<40 \mathrm{mg} / \mathrm{dL}), \geq 1.6 \mathrm{mmol} / \mathrm{L}$ $(\geq 60 \mathrm{mg} / \mathrm{dL})$ counts as a "negative" risk factor; its presence removes 1 risk factor from the total count. Family history of premature coronary heart disease: CHD in male first-degree relative $<55$ years and $\mathrm{CHD}$ in female first-degree relative $<65$ years. Age and Lifestyle risk factors like Obesity (body mass index $\geq 30 \mathrm{~kg} / \mathrm{m}^{2}$ )

\section{STATISTICAL ANALYSIS}

The mean level of serum uric acid levels under different categories of CAD risk factors and different categories of coronary angiography was compared using unpaired student t-test.

For the statistical significance of different categories of serum uric acid levels (normal and abnormal) with different categories of CAD risk factors and coronary angiography chi-square /Fischer's exact test was applied. Kruskal-Wallis Test was applied for the statistical significance of uric acid with different age groups. $\mathrm{p}<0.05$ was taken as level of statistical significance. The relationship between serum lipid and serum uric acid levels was calculated using Pearson's correlation co-efficient. The data was analyzed using SPSS statistical software version 16.

\section{RESULTS}

A total of 100 patients diagnosed with ACS (Acute Coronary Syndrome) satisfying all inclusion and exclusion criteria as mentioned in aims and objectives were included in this study. Study parameters included the serum uric acid, CAD risk factors and coronary angiographic findings. The results were analysed in accordance with the aims and objectives of our study.

It was observed that 33\% patients had STEMI, 44\% had diagnosis of NSTEMI and 23\% patients had diagnosis of UNSTABLE ANGINA. Of the study population $76 \%$ were

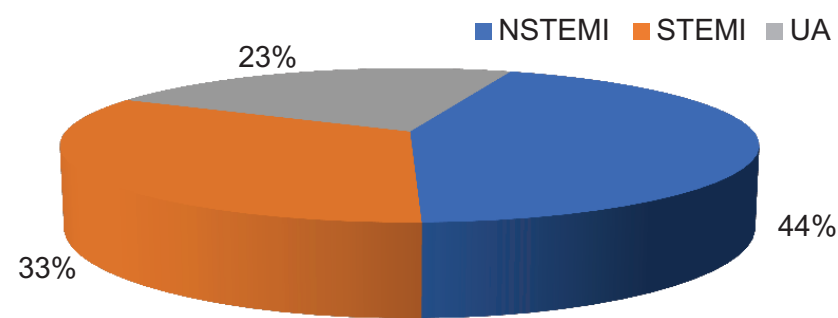

Figure-1: Composition of patients with acute coronary syndrome

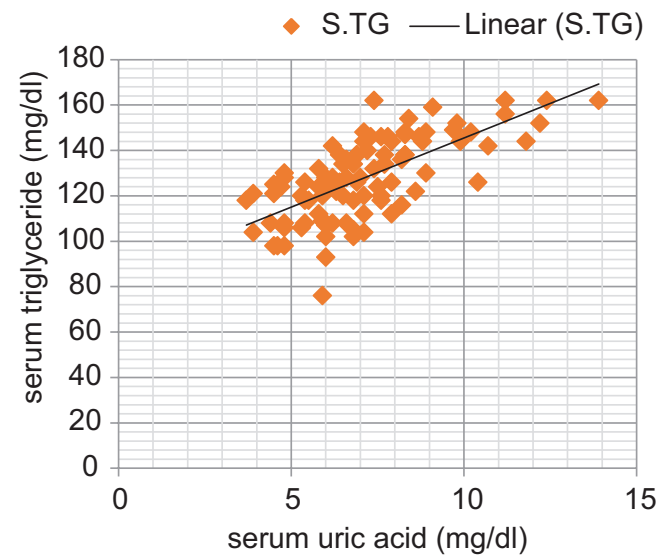

Figure-2: demonstrates a significant positive correlation between the serum triglyceride and serum uric acid. (Pearson correlation coefficient $(\mathrm{r})=0.698, \mathrm{p}<0.001)$ 


\begin{tabular}{|c|c|c|c|c|c|}
\hline Variables & & $\begin{array}{l}\text { Mean serum uric } \\
\text { acid } \pm S D(m g / d l)\end{array}$ & Non-hyperuricemia & Hyper Uricemia & $P$ value \\
\hline \multirow[t]{2}{*}{ Hyper Tension } & $\begin{array}{l}\text { Yes } \\
N=60\end{array}$ & $7.665 \pm 2.01$ & 19/60 (31.7) & 41/60(68.3) & \multirow[t]{2}{*}{.001} \\
\hline & $\begin{array}{l}\text { No, } \\
N=40,\end{array}$ & $6.292 \pm 1.66$ & $26 / 40(65)$ & $14 / 40(35)$ & \\
\hline \multirow[t]{2}{*}{ Diabetes mellitus } & $\begin{array}{l}\text { Yes, } \\
\mathrm{N}=45\end{array}$ & $7.769 \pm 2.14$ & $13 / 45(28.9)$ & $32 / 45(71.1)$ & \multirow[t]{2}{*}{.003} \\
\hline & $\begin{array}{l}\text { No, } \\
\mathrm{N}=55\end{array}$ & $6.57 \pm 1.66$ & $32 / 55(58.2)$ & $23 / 55(41.8)$ & \\
\hline \multirow[t]{2}{*}{ DYS Lipidemia } & $\begin{array}{l}\text { Yes, } \\
N=65,\end{array}$ & $7.683 \pm 2.07$ & $22 / 65(33.8)$ & 43/65(66.2) & \multirow[t]{2}{*}{.002} \\
\hline & $\begin{array}{l}\mathrm{No} \\
\mathrm{N}=35\end{array}$ & $6.05 \pm 1.21$ & $23 / 35(65.7)$ & $12 / 35(34.3)$ & \\
\hline
\end{tabular}

\begin{tabular}{|c|c|c|c|c|c|c|}
\hline Risk factor & & $\begin{array}{c}\text { Mean } \\
\text { SUA } \pm S D \\
\text { (mg/dl) }\end{array}$ & $\begin{array}{c}\text { Hyper } \\
\text { uricemia }\end{array}$ & $\begin{array}{l}\text { Non-hyper } \\
\text { uricemia }\end{array}$ & $P$ value & Significance \\
\hline \multirow[t]{2}{*}{ Family history of premature CHD } & $\begin{array}{l}\text { Yes, } \\
N=44\end{array}$ & $7.157 \pm 2.25$ & $21 / 44(47.7)$ & $23 / 44(52.27)$ & \multirow[t]{2}{*}{0.627} & \multirow[t]{2}{*}{ NS } \\
\hline & $\begin{array}{l}\text { No, } \\
N=56\end{array}$ & $7.073 \pm 1.74$ & $24 / 56(42.9)$ & $32 / 56(57.1)$ & & \\
\hline \multirow[t]{2}{*}{ Smoking } & $\begin{array}{l}\text { Yes, } \\
N=62\end{array}$ & $6.887 \pm 1.728$ & $31 / 62(50)$ & $31 / 62(50)$ & \multirow[t]{2}{*}{0.199} & \multirow[t]{2}{*}{ NS } \\
\hline & $\begin{array}{l}\mathrm{No}, \\
\mathrm{N}=38\end{array}$ & $7.474 \pm 2.299$ & $14 / 38(36.8)$ & $24 / 38(63.2)$ & & \\
\hline \multirow[t]{2}{*}{ BMI } & $\begin{array}{l}\text { Normal (18.5-24.9), } \\
\mathrm{N}=80\end{array}$ & $7.082 \pm 1.86$ & $36 / 80(45)$ & $44 / 80(55)$ & \multirow[t]{2}{*}{0.65} & \multirow[t]{2}{*}{ NS } \\
\hline & $\begin{array}{l}\text { Over weight } \\
(>25) \\
N=20\end{array}$ & $7.220 \pm 2.4$ & $9 / 20(45)$ & $11 / 20(55)$ & & \\
\hline
\end{tabular}

\begin{tabular}{|l|c|c|c|c|}
\hline Coronary angiographic findings & Mean SUA \pm SD (mg/d) & Hyperuricemia & Non-hyperuricemia & P value \\
\hline Absent, $n=1$ & 3.7 & $0 / 1(0)$ & $1 / 1(100)$ & $<0.001$ \\
\hline 1 vessel, $n=26$ & $5.58 \pm 1.14$ & $6 / 26(23.1)$ & $20 / 26(76.9)$ \\
\hline 2 vessel, $n=54$ & $6.85 \pm 1.05$ & $30 / 54(55.6)$ & $24 / 54(44.4)$ \\
\hline 3 vessel, $n=19$ & $10.11 \pm 1.63$ & $19 / 19(100)$ & $0(0)$ & \\
\hline \multicolumn{4}{|r|}{ Table-3: Comparison of coronary angiographic findings between the two groups } \\
\hline
\end{tabular}

males and 24\% females. Males were more than females in all the categories of acute coronary syndrome. It was found that around $13 \%$ of patients were in the age group $<40 \mathrm{yrs}$. This shows that ACS is emerging among people in their mid and late thirties. Majority of the patients were in the age group of 40 to $60 \mathrm{yrs}(56 \%)$. The elderly age group i.e above $65 \mathrm{yrs}$ comprised $31 \%$ of the ACS patients.

In the study, $55 \%$ of the population had hyperuricemia and the mean serum uric acid $(\mathrm{mg} / \mathrm{dl})$ was $7.11 \pm 1.97$. The serum uric acid levels and their association with each CAD risk factor was assessed. It was found that the the percentage of patients with hyperuricemia increases with age however, this association is not statistically significant $(p=0.258)$.[Figure 3]

As shown in the table 1 , there was no stastically significant difference with regards to age and sex in the uric acid levels of ACS patients. Though $66.7 \%$ of females had hyperuricemia as compared to males (51.3\%), this difference was not significant $(\mathrm{p}=0.188)$. Similarly, there was no significant association between age as a CAD risk factor and uric acid. $(\mathrm{p}=0.258)$.

The mean serum uric acid of hypertensives in the study was $7.665 \pm 2.001$. and that of non hypertensives is $6.292 \pm 1.66$ and this difference was statistically significant. There was significant association between higher serum uric acid level and patients who were hypertensive $(p<0.05)$. Similarly, mean serum uric acid in the diabetics in the study was $7.769 \pm 2.137$ and that of non-diabetics was $6.57 \pm 1.66$. The difference between the two groups was statistically significant $(p=0.003)$. Hence, there is a significant association 


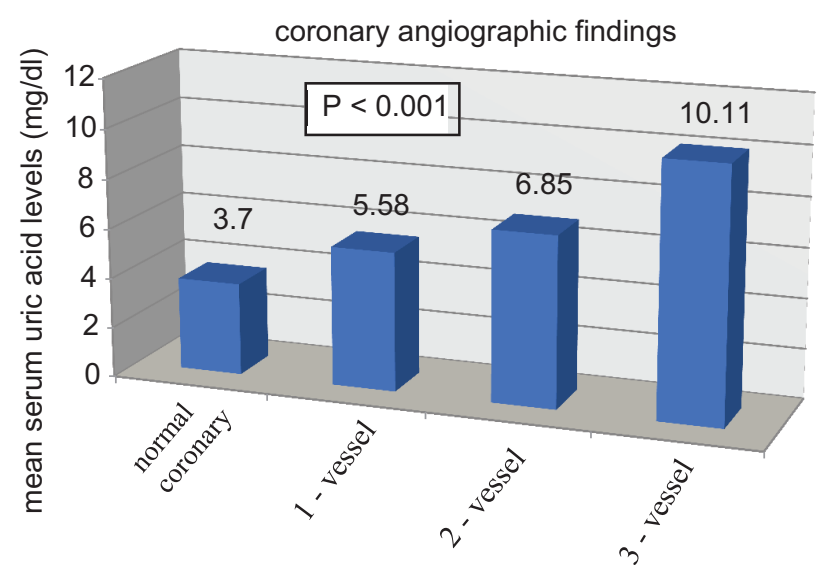

Figure-3: shows that the severity of the CAD lesions (based on the number of vessels involved) increases with an increase in the mean serum uric acid levels.

between uric acid and the diabetic status. Hypertensives in the study group had a higher mean serum uric acid level compared to the non-hypertensive group.

Association between serum uric acid levels and other CAD risk factors were also looked into. There was no statistically significant association between uric acid and Family history of premature CHD ( $p=0.627)$. There was no statistically significant difference in the mean serum uric acid of smokers and non-smokers $(p=0.199)$. Similarly, there was no significant association between uric acid and BMI of the patient $(\mathrm{p}=0.65)$. There was a significant positive correlation between SUA and TRIGLYCERIDES $(r=0.698, p<0.001)$ and a negative correlation between SUA and LDL Cholesterol. ( $r=.078, \mathrm{p}>0.01)$. demonstrates a significant positive correlation between the serum triglyceride and serum uric acid. (Pearson correlation coefficient $(r)=0.698$, $\mathrm{p}<0.001)$.

The patients in the hyperuricemia group had more number of coronary vessels involved than the other group. It was found that patients with no significant coronary lesions had a mean serum uric acid level of $3.7 \mathrm{mg} / \mathrm{dl}$, whereas those with 1 vessel, 2 - vessel and 3-vessel involvement had a mean serum uric acid level of 5.58,6.85 and $10.11 \mathrm{mg} / \mathrm{dl}$ respectively. The difference in the mean serum uric acid level was significantly associated with the severity of CAD ( $\mathrm{p}<0.001)$. Hence, it was found that increase in serum uric acid level was associated with an increase in the severity of the CAD lesions in the patients with acute coronary syndrome.

\section{DISCUSSION}

Unlike many disabling medical conditions, cardiovascular disease which is already one of the the most common cause of deaths - is almost entirely preventable. Acute coronary syndrome is the most dramatic and serious consequence of cardiovascular disease.

Our study consisted of 100 ACS patients (including Unstable Angina, NSTEMI and STEMI). The mean serum uric acid in the patients was $7.11 \pm 1.97 \mathrm{mg} / \mathrm{dl}$. There have been a few studies in the recent times assessing the clinical value of SUA in acute myocardial infarction, however very few have assessed its value in the whole spectrum of ACS patients and assessed its correlation with severity of CAD.
In the Japanese Acute Coronary Syndrome study, there was a correlation between SUA concentration and Killip classification in patients with acute MI patients. Patients who developed short term adverse effects have high SUA concentration. ${ }^{8}$ Similar findings were also seen in a study by Nadkar et al, conducted in 100 patients of acute MI, SUA levels were higher in acute MI patients compared to normal healthy persons. ${ }^{9}$ In another study by M Baruah et al, there was a statistically significant level of SUA in patients of Acute MI on day 1,3 and 5 and at time of discharge as compared to controls $(\mathrm{p}<.001) .{ }^{10}$

We also evaluated the correlation of serum uric acid with various CAD risk factors in the selected ACS patients. We did not find any significant association between SUA and gender of the patient $(p=0.188)$ However, Culeton et al, had reported in a study concerning role of uric acid as an independent risk factor in CAD, that they found an increased risk for adverse outcome after age adjustment only for women. ${ }^{3}$ In a study by Zhang et al, it was found that the SUA levels were significantly associated with the prevalence of CAD and that SUA levels may be a predictor for incidence of major cardiovascular events in premenopausal women. ${ }^{11}$ Age as a CAD risk factor was also not significantly associated with SUA concentration in our study. $(p=0.191)$

In our study there was a significant relation $(p=0.001)$ between serum uric acid level and patients who were known or found to be hypertensive on admission. (Table 3 and Figure 3) Our study showed that hypertensive patients had more hyperuricemia. Similar findings were also seen in a study by Schmidt et al, where compared with normotensive patients SUA levels were significantly elevated in hypertensive patients. ${ }^{12}$ The Framingham Heart Study also revealed that serum uric acid was independent predictor of presence and progression of hypertension. In a study by Kojima et al, assessing the prognostic value of serum uric acid in ACS patients, it was noted that SUA concentration was significantly correlated with hypertension. $(r=0.301$, $\mathrm{p}=.005) .{ }^{8}$ Indeed, the US NHANES iii showed that ageadjusted rates of myocardial infarction are higher across increasing serum uric acid quartiles among male and female hypertensive patients. ${ }^{13}$

$45 \%$ of the patients in our study were diabetic. Figure 4 and table 3 show that hyperuricemia is significantly associated with diabetes mellitus $(\mathrm{p}=.003)$. This is in accordance with a study by Perry et al [1995], which concluded that elevated serum uric acid concentration predicts subsequent development of diabetes mellitus, even in the presence of normal creatinine clearance and plasma glucose concentration, and therefore may be a subtle early marker of peripheral insulin resistance syndrome. ${ }^{14}$

Our study found that there was a significant association between serum uric acid and dyslipidemia $(p=0.002)$ and previous history of coronary artery disease $(p=0.004)$. In this study, SUA levels were positively correlated with serum triglyceride level $(\mathrm{r}=0.698, \mathrm{p}<.001)$. In the Coronary Artery Risk Development in Young Adults (CARDIA) study in 4043 white and black men, an independent relationship between hyperuricemia and triglycerides was reported. ${ }^{15}$ Desai et al, have also shown a linear and independent 
association between serum uric acid levels and the TG/ HDL- Cholesterol ratio. ${ }^{16}$ The mechanism of the close relationship between SUA level and lipid metabolism has not been understood completely. It may involve both metabolic and genetic defects, which mean lipid metabolic disorders along with purine metabolic disorders. Meanwhile afferent and efferent arterioles of glomerulus can be involved when lipid metabolic disorders occur, resulting in the stenosis or occlusion of arteries and transrenal excretion of uric acid. Chen et al, in their study in 502 patients of STEMI found that serum uric acid was positively correlated with serum triglyceride level. Hyperlipidemia was more common in hyperuricemia patients than in non-hyperuricemia patients (43.7\% vs $33.7 \%, p=0.014)$, and serum triglyceride level was significantly higher in hyperuricemia patients $(2.11 \pm 1.24$ vs $1.78 \pm 1.38, \mathrm{p}=0.014) \mathrm{mmol} / \mathrm{L} .{ }^{17}$

In the present study, we did not find any statistically significant difference $(p=0.65)$ in the difference in the mean serum uric acid of our patients with normal BMI and those with a BMI > 25. However, in a study by Madani et al, it was reported that SUA levels were positively correlated with BMI. ${ }^{18}$ We also found no significant association between SUA and smoking $(\mathrm{p}=0.199)$ and family history of premature coronary heart disease $(p=0.627)$.

Uric acid promotes the development of atherosclerosis, while causing a variety of cardiovascular events. The possible mechanisms vary. The high level of SUA promotes the oxidation of LDL-C and the peroxidation of lipid. It also increases the formation of oxygen radicals in inflammatory reaction. In addition, the high level of SUA also increases platelet aggregation and the formation of uric acid crystals. The deposition of uric acid in the arterial wall could damage the tunica intima of arteries, promoting coronary thrombosis. By investigating our patients undergoing coronary angiography, we found that hyperuricemia was associated with the presence of angiographic documentation of CAD in the whole population of ACS patients who were studied. Moreover, we found that there was an increase in the severity of CAD scaled by clinical vessel score with the increase in the SUA levels $(\mathrm{p}<0.001)$.

In a study by Goodarzynejad et al, in 370 patients having angiographic evidence of atherosclerosis, it was shown that asymptomatic hyperuricemia may be associated with the presence and severity of angiographically defined CAD. (adjusted OR $=1.51,95 \% \mathrm{CI}=1.09-2.09, \mathrm{p}=0.005) .{ }^{19} \mathrm{In}$ a Tuttle and colleagues study, there was also a linear correlation between uric acid levels and CAD severity in women but not in men. ${ }^{20}$ In a study in Turkey, Deveci et al, evaluated 1012 patients for association of serum uric acid with angiographic presence and severity of IHD using Gensini score. A statistically significant difference in the mean uric acid concentrations was found between the patients with and without coronary artery disease irrespective of sex. ${ }^{21}$

However, in a study by Chen et al, it was seen that serum uric acid in 502 patients of acute STEMI, positively correlated with the presence but not the severity of CAD ( $>0.05)$. 17 Similarly, in a study by Gur M et al,in evaluating the relationship between serum uric acid and the severity of $\mathrm{CAD}$ assessed by the Gensini score, there was no significant correlation between UA levels and Gensini score.( $r=0.083$. $\mathrm{p}>0.05) .{ }^{22}$ Similarly, an Indian study by Gangadharam et al, concluded that serum UA level in patients with acute coronary syndromes was not associated with Killip class and extent of coronary vessels involved. ${ }^{23}$

\section{CONCLUSION}

Serum uric acid is elevated in patients with acute coronary syndrome. There is a positive correlation between serum uric acid and CAD risk factors like hypertension and diabetes.

Dyslipidemia and previous history of CAD were associated with elevated serum uric acid levels. The serum uric acid level is correlated with the presence and severity of CAD by coronary angiographic findings $(\mathrm{p}<0.001)$.

\section{CONCLUSION}

Uric acid being an inexpensive and easily available biochemical marker, it will be of much use in settings with limited resources. Compared to markers such as Brain Natriuretic peptide (BNP) and hs CRP which have been widely used in assessing prognosis of acute MI, whether SUA could be an independent predictor of prognosis of the patients with ACS needs to be confirmed by further studies. Serum uric acid can also be used to predict the severity of CAD. Hence, uric acid should form an integral part of the work up of the management of ACS patients. More interventional studies concerning the lowering of SUA levels and their benefit on cardiovascular related mortality in ACS patients need to be carried out.

\section{REFERENCES}

1. Kolansky DM. Acute coronary syndromes: morbidity, mortality, and pharmacoeconomic burden. Am J Manag Care. 2009;15(suppl):S36-41.

2. de Araújo Gonçalves P, Ferreira J, Aguiar C, SeabraGomes R. TIMI, PURSUIT, and GRACE risk scores: sustained prognostic value and interaction with revascularization in NSTE-ACS. Eur Heart J. 2005; (26):865-72.

3. Culleton BF, Larson MG, Kannel WB, Levy D. Serum uric acid and risk for cardiovascular disease and death: the Framingham Heart Study. Ann Intern Med. 1999;(131):7-13.

4. Anker SD, Doehner W, Rauchhaus M, Sharma R, Francis D, Knosalla C. Uric acid and survival in chronic heart failure: validation and application in metabolic, functional, and hemodynamic staging. Circulation. 2003;(107):1991-97.

5. Franse LV, Pahor M, Di Bari M, Shorr RI, Wan JY, Somes GW. Serum uric acid, diuretic treatment and risk of cardiovascular events in the Systolic Hypertension in the Elderly Program (SHEP). J Hypertens. 2000;(18):1149-54.

6. Lehto S, Niskanen L, Rönnemaa T, Laakso M. Serum uric acid is a strong predictor of stroke in patients with non-insulin-dependent diabetes mellitus. Stroke. 1998;(29):635-39.

7. Bickel C, Rupprecht HJ, Blankenberg S, Rippin G, Hafner G, Daunhauer A. Serum uric acid as an independent predictor of mortality in patients with 
angiographically proven coronary artery disease. Am J Cardiol. 2002;(89):12-17.

8. Kojima S, Sakamoto T, Ishihara M, et al. Prognostic usefulness of serum uric acid after acute myocardial infarction (Japanese Acute Coronary Syndrome Study). Am J Cardiol 2005;96(2):489-95.

9. Nadkar MY, Jain VI. Serum Uric Acid in Acute Myocardial Infarction. JAPI.2008;56(1):759-62.

10. Baruah M, Nath CK, Chaudhury M, Devi R, Shaker A, Ivvala. A Study of Serum Uric Acid and C-Reactive Protein in Acute Myocardial Infarction. International J of Basic Medical Sciences and Pharmacy. 2012; 2(1):2124.

11. Zhang J-w, He L-j, Cao S-j, Yang Q, Yang S-w, et al. (2014) Association of Serum Uric Acid and Coronary Artery Disease in Premenopausal Women. PLoS ONE 9(9): e106130.

12. SchmidtMI, Watson RL,Duncan BB, et al. Clustering of dyslipidemia, hyperuricemia, diabetes, and hypertension and its association with fasting insulin and central and overall obesity in a general population. Atherosclerosis Risk in Communities Study Investigators. Metabolism. 1996;45(3):699-706.

13. Liese AD, Hense HW, Lowel H, Doring A, Tietze M, Keil U. Association of serum uric acid with allcause and cardiovascular disease mortality and incident myocardial infarction in the MONICA Augsburg cohort. World Health Organization Monitoring Trends and Determinants in Cardiovascular Diseases. Epidemiology1999; 10(6):391-7.

14. Perry IJ, Wannamethee SG, Walker MK, Thomson AG, Whincup PH, Shaper AG. Prospective study of risk factors for development of non-insulin dependent diabetes in middle aged British men. BMJ 1995; 310(4):560-4.

15. Rathman W, Funkhouser E, Dyer AR, Roseman JM. Relations of hyperuricemia with the various components of the insulin resistance syndrome in young black and white adults: the CARDIA study. Coronary Artery Risk Development in Young Adults. Ann Epidemiol 1998;8(2):250-61.

16. Desai MY, Dalal D, Santos RD, et al. Association of body mass index, metabolic syndrome, and leukocyte count. Am J Cardiol 2005;97(1):835-8.

17. Chen L, Li X, Qiao W, Ying Z, Qin Y, Wang Y,et al. Serum uric acid in patients with acute ST-elevation myocardial infarction. World J Emerg Med. 2012; $3(1): 35-9$

18. Madani MK, Abdrabo AA. Association between serum uric acid and selected components of metabolic syndrome. Laboratory med J 2013: 1(1):23-29.

19. Goodarzynejad H, Anvari MS, Boroumand MA, Karimi A, Abbasi HA, Davoodi.Hyperuricemia and the Presence and Severity of Coronary Artery Disease. Labmedicine.2010;41(1):40-45.

20. Tuttle KR, Short RA, Johnson RJ. Sex differences in uric acid and risk factor for coronary artery disease. Am J Cardiol. 2001;87(4):1411-1414.

21. Deveci OS, Kabakci G, Okutucu S, Tulumen E, Aksoy $\mathrm{H}$, Kaya EB, et al. The association between serum uric acid level and coronary artery disease. Int J Clin Pract.
2010;64(7):900-907.

22. Gur M, yilmaz R, Demirbag $R$ et al. Relation of serum uric acid levels with the presence and severity of angiographic coronary artery disease. Angiology 2008;59(6): 166-71.

23. Y. Gangadharam, Noushadali. Serum uric acid level is not associated with severity and extent of coronary disease in patients with acute coronary syndromes - Our experience. IAIM, 2017; 4(1): 44-49.

\section{Source of Support: Nil; Conflict of Interest: None}

Submitted: 11-09-2019; Accepted: 16-11-2019; Published online: 12-12-2019 